SCIENTIFIC LETTER

\title{
Vitamin $C$ is not associated with coronary heart disease risk once life course socioeconomic position is taken into account: prospective findings from the British women's heart and health study
}

\author{
D A Lawlor, S Ebrahim, D Kundu, K R Bruckdorfer, P H Whincup, G Davey Smith
}

Heart 2005;91:1086-1087. doi: 10.1136/hrt.2004.048934

$\mathrm{T}$ he reason why prospective cohort studies have found that plasma vitamin $C$ concentration, or dietary intake of vitamin $C$, is protective against coronary heart disease (CHD) risk ${ }^{1}$ but randomised controlled trials have found no effect of vitamin $\mathrm{C}$ on $\mathrm{CHD}^{2}$ is unclear. We have previously suggested that this difference is caused by confounding. ${ }^{3}$ Our hypothesis is that high adult concentrations of vitamin $\mathrm{C}$ reflect a number of characteristics, including advantageous socioeconomic circumstances in childhood and adulthood, and that it is these characteristics, not vitamin $\mathrm{C}$, that protect against CHD. The aim of this study is to examine whether the association between plasma vitamin $\mathrm{C}$ and CHD is confounded by life course socioeconomic position.

\section{METHODS}

Data from the British Women's Heart and Health Study were used. Full details of the selection of participants and measurements used in the study have been previously reported. ${ }^{4}$ Between 1999 and 2001, 4286 British women aged 60-79 years were interviewed, examined, completed medical questionnaires, and had detailed reviews of their medical records. These women have been followed up over a median of four years by flagging with the National Health Service (NHS) central register for mortality data and two yearly reviews of their medical records. This follow up is complete for all participants. Local ethics committee approval was obtained for the study.

Ten measures of life course socioeconomic position were obtained from baseline questionnaire responses: father's occupational social class, bathroom and hot water in childhood home, family car access, own bedroom, age at leaving full time education, husband's/own occupational social class, adult housing tenure, adult car access, and pension arrangements. ${ }^{4}$ Standard methods for assessing vitamin $\mathrm{C}$ were used (details available from authors). Incident cases of CHD in women who were free of prevalent CHD at baseline were defined as either: death with an underlying or contributing cause of CHD (International classification of diseases, 10 revision (ICD-10) codes I20-I25, I51.6), or a diagnosis of myocardial infarction (fulfilling World Health Organization criteria), angina, coronary artery bypass, or angioplasty identified from the medical record review. Patients were censored at the occurrence of death from any other cause or when 1 June 2004 was reached. Cox proportional hazards models were used to assess the association between vitamin $\mathrm{C}$ and incident cases of CHD. Age was used as the time scale, and visual inspection of survival curves demonstrated that the assumption of proportionality was not violated in any models. All analyses were conducted using Stata version 8.0.

\section{RESULTS}

Of the 4286 women studied, 3659 (85\%) had adequate vitamin assays and details of all indicators of socioeconomic position. The prevalence of CHD at baseline was similar in those with and without these data $(\mathrm{p}=0.23)$. Of the 3659 women with complete data 586 (16\%) had CHD at baseline; these were excluded from further analyses. Among the remaining 3073 women, there were 158 new cases of CHD over 12893 person years of follow up, giving a rate of 12.9 (95\% CI 10.5 to 14.3 ) per 1000 person years. There was a linear decrease in mean vitamin $C$ with each additional adverse life course socioeconomic indicator (for linear association, $\mathrm{p}<0.001$, and for non-linear association, $\mathrm{p}=0.8$ ) (fig 1). CHD increased linearly with each additional adverse socioeconomic position indicator (linear trend, $\mathrm{p}=0.001$, and non-linear association, $\mathrm{p}=0.5$ ). However, because of the small number of incident cases in some categories we collapsed the life course socioeconomic position score into two categories. Among those with 0-4 adverse socioeconomic indicators the rate of CHD was 13.2 (95\% CI 8.1 to 12.9 ) per 1000 person years and among those with 5-10 adverse indicators it was 15.2 (95\% CI 12.3 to 18.8), p for difference $=0.03$.

We used a measure of association with vitamin C to match that achieved by supplementation in the Heart protection randomised controlled trial (relative risk per $15.7 \mu \mathrm{mol} / \mathrm{l}$ ). ${ }^{2}$ The hazard ratio (HR) of a new CHD event per $15.7 \mu \mathrm{mol} / \mathrm{l}$

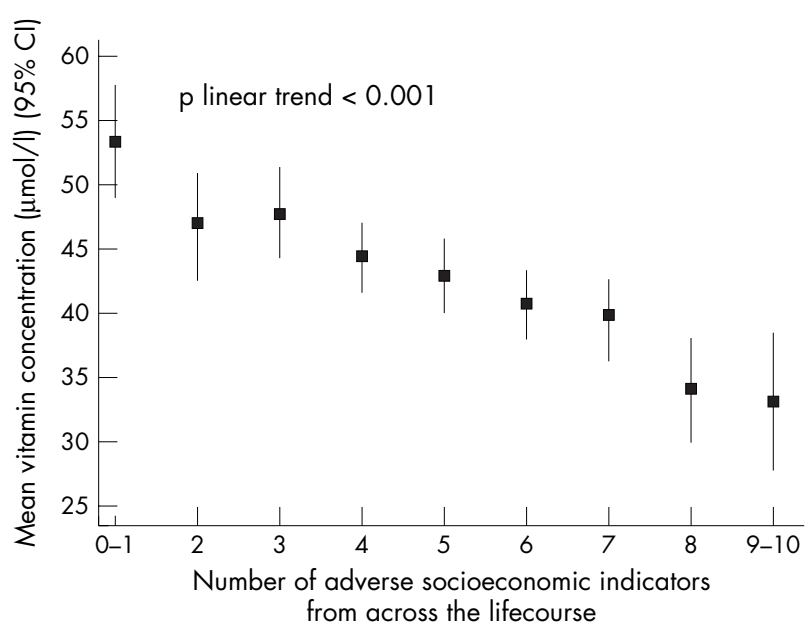

Figure 1 Mean vitamin $C$ concentrations by number of adverse life course socioeconomic position indicators among British women aged $60-79$ years. 
increase in plasma vitamin C concentration was 0.88 (95\% CI 0.80 to 0.97 ). Adjustment for the same list of risk factors used in the EPIC prospective cohort study (systolic blood pressure, cholesterol, body mass index, cigarette smoking, diabetes and vitamin supplement use) attenuated this association to 0.90 (95\% CI 0.82 to 0.99). Addition to this model of other potential adult confounders (physical activity, alcohol consumption, fruit and vegetable consumption, adult social class) did not result in any further attenuation. When we adjusted only for number of adverse life course socioeconomic indicators the crude association attenuated from 0.88 to 0.92 ( $95 \%$ CI 0.83 to 1.01). Full adjustment for life course socioeconomic position, adult risk factors, leg length and forced expiratory volume in one second resulted in a hazard ratio of 0.95 (95\% CI 0.85 to 1.05). A stratified analysis found that the hazard ratio among those with $0-4$ adverse indicators was 0.89 (95\% CI 0.76 to 1.02) and among those with 5-10 adverse indicators it was 0.95 (95\% CI 0.83 to $1.09)$.

\section{DISCUSSION}

Mean plasma vitamin $\mathrm{C}$ decreases with each additional adverse life course socioeconomic position indicator, whereas CHD risk increases with worsening life course socioeconomic position. Consistent with previous observational studies we found that higher concentrations of vitamin C were associated with lower CHD risk. ${ }^{1}$ However, our association was weaker than that reported in previous studies and it is possible that previous observational studies with weak or null effects for this association have failed to be published (publication bias). When life course socioeconomic position was taken into account, either in multivariable models or by stratified analysis, there was no association between vitamin $\mathrm{C}$ and CHD.

Among participants with no CHD at baseline there were just 158 new cases. Removal of those with baseline CHD is important to ensure that associations are not caused by reverse causality. Our study includes more cases of CHD than were included for both women and men in the EPIC cohort study. ${ }^{1}$ We have used retrospective self report of childhood socioeconomic status, but any measurement error is likely to be non-differential and would therefore tend to underestimate the true confounding effect of life course socioeconomic position. ${ }^{5}$

Our results suggest that previous observational studies reporting a protective effect of vitamin $\mathrm{C}$ may have been influenced by publication bias and confounding caused by the failure of the investigators to appreciate that adult plasma vitamin $\mathrm{C}$ concentrations are influenced by socioeconomic position from across the life course. Our results are supportive of randomised controlled evidence that there is no important causal association between vitamin $C$ and coronary heart disease. We discuss elsewhere how observational epidemiology can be improved to avoid this problem. ${ }^{3}$

\section{ACKNOWLEDGEMENTS}

We thank all of the women who participated in the study, their general practitioners who supported data collection, and Rita Patel, Carol Bedford, Alison Emerton, Nicola Frecknall, Karen Jones, Mark Taylor, Simone Watson, and Katherine Wornell for collecting and entering data.

The British Women's Heart and Health Study is funded by the Department of Health, and the British Heart Foundation provided funds for the vitamin C assays. DAL is funded by a Department of Health Career Scientist Award. The views expressed in this publication are those of the authors and not necessarily those of any funding body.

\section{Authors' affiliations}

D A Lawlor, S Ebrahim, G Davey Smith, Department of Social Medicine, University of Bristol, Bristol, UK

D Kundu, K R Bruckdorfer, Department of Biochemistry and Molecular Biochemistry, Royal Free Hospital School of Medicine, University of London, London, UK

P H Whincup, Department of Community Health Sciences, St George's Hospital Medical School, University of London

Conflict of interest: none

Correspondence to: Dr Debbie A Lawlor, Department of Social Medicine, University of Bristol, Canynge Hall, Whiteladies Road, Bristol, BS8 2PR, UK; d.a.lawlor@bristol.ac.uk

Accepted 25 October 2004

\section{REFERENCES}

1 Khaw KT, Bingham S, Welch A, et al. Relation between plasma ascorbic acid and mortality in men and women in EPIC-Norfolk prospective study: a prospective population study. European prospective investigation into cancer and nutrition. Lancet 2001;357:657-63.

2 Heart Protection Study Collaborative Group. MRC/BHF heart protection study of antioxidant vitamin supplementation in 20536 high-risk individuals: a randomised placebo-controlled trial. Lancet 2002;360:23-33.

3 Lawlor DA, Davey Smith G, Bruckdorfer KR, et al. Those confounded vitamins: what can we learn from the differences between observational versus randomised trial evidence? Lancet 2004;363:1724-7.

4 Lawlor DA, Bedford C, Taylor M, et al. Geographic variation in cardiovascular disease, risk factors and their control in older women: British women's heart and health study. J Epidemiol Community Health 2003;57:134-40.

5 Davey Smith G, Lynch J. Life course approaches to socioeconomic differentials in health. In: Kuh D, Ben-Shlomo Y. A life course approach to chronic disease epidemiology, 2nd ed. Oxford: Oxford University Press, 2004:77-115. 